\title{
Effects of early treatment with zofenopril in patients with myocardial infarction and metabolic syndrome: the SMILE Study
}

\author{
Claudio Borghi \\ Arrigo FG Cicero \\ Ettore Ambrosioni \\ Department of Clinical Medicine, \\ University of Bologna, Bologna, Italy. \\ On behalf of the Survival \\ of Myocardial Infarction Long-term \\ Evaluation (SMILE) Study
}

\begin{abstract}
Objective: To evaluate the clinical efficacy of the early administration of zofenopril in a group of patients with and without metabolic syndrome (MS+ and MS-) and anterior myocardial infarction enrolled in the Survival of Myocardial Infarction Long-Term Evaluation (SMILE) Study.

Methods: Patients were randomized double-blind to zofenopril $(n=719)$ or placebo $(n=699)$ for 6 weeks. The primary end point was the effect of treatment on the 6-week combined occurrence of death and severe congestive heart failure. The secondary end point was the 1-year mortality rate.

Results: Of the 1418 patients included in this post-hoc analysis, 686 (48.3\%) had MS. After 6 weeks of treatment zofenopril significantly reduced the incidence of all-cause death and severe congestive failure (risk reduction: $69 \%, 95 \% \mathrm{CI}: 7-78 ; 2 \mathrm{p}=0.002$ ) in MS+ patients. This was the case for 1-year mortality, too $(29 \%, 95 \% \mathrm{CI}: 4-41 ; 2 \mathrm{p}=0.048)$. Zofenopril was effective also in MS- patients but the amount of relative risk reduction was less than in MS+ for both the primary $(-11 \% ; 2 p=0.61)$ and secondary endpoint $(-19 \% ; 2 p=0.025)$.
\end{abstract}

Conclusions: Results of this post-hoc analysis of the SMILE Study demonstrate the striking benefit of early administration of zofenopril in MS+ patients with acute anterior myocardial infarction.

Keywords: SMILE Study, angiotensin converting enzyme inhibitor, zofenopril, myocardial infarction, metabolic syndrome

\section{Introduction}

Early treatment with angiotensin-converting enzyme (ACE) inhibitors of patients with acute myocardial infarction greatly improves their in-hospital and long-term survival (Borghi et al 1998; Donnelly and Manning 2007). Most of the benefit of ACE inhibitors in these patients is due to blockade of both plasma and tissue renin-angiotensin system, activated during the early phase of myocardial infarction (Omland et al 1993; Walsh et al 1999).

Metabolic syndrome (MS) is a constellation of cardiovascular risk factors, such as abdominal obesity, atherogenic dyslipidemia, hypertension, and insulin resistance or glucose intolerance (Eckel et al 2005), and it is associated with an increased cardiovascular morbidity and mortality (Hu et al 2004; Hunt et al 2004). MS has a prevalence of $15 \%-20 \%$ in the general population (Ford et al 2002) but it occurs in nearly $50 \%$ of unselected patients with acute myocardial infarction (Zeller et al 2005). In these patients MS further enhances the risk of death from all causes as well as the risk of cardiovascular complications (Ninomiya et al 2004; Levantesi et al 2005). Recent evidence supports the hypothesis that the hyperinsulinemia that characterizes the subjects with MS may be associated with an overexpression of vascular angiotensin 
II-AT ${ }_{1}$ receptors leading to an exaggerated activation of the tissue renin-angiotensin system, a condition particularly harmful in patients with acute myocardial infarction (Muller et al 2000; Zhang et al 2005). For this reason ACE inhibition may play a key role in patients with myocardial infarction and MS (Prasad and Quyyumi 2004).

Zofenopril calcium is a sulfhydryl ACE inhibitor (Borghi et al 2004) which has been shown in the SMILE (Survival of Myocardial Infarction Long-term Evaluation) Study to improve both short- and long-term outcome when administered within the first 24 hours of an acute myocardial infarction (Ambrosioni et al 1995). The present study is a post-hoc analysis of the SMILE Study to evaluate the effects of the early administration of zofenopril on the clinical outcome in a subgroup of patients with (MS+) and without MS (MS-).

\section{Material and methods}

\section{Subjects}

The SMILE Study included 1556 patients admitted to 154 Italian coronary care units who were randomized to study treatment with an ACE inhibitor or placebo in addition to recommended pharmacological treatment. Details of the study protocol have been published elsewhere (Ambrosioni et al 1995). In the main SMILE Study, eligible patients were considered those of either gender, aged 18-80 years, presenting to the intensive care unit within 24 hours of the onset of chest pain typically associated with electrocardiographic signs of myocardial infarction of the anterior wall and not eligible for thrombolytic therapy because of late admission to the intensive care unit or contraindications to systemic fibrinolysis. Upon enrollment patients were randomly allocated according to fixed blocks to receive oral zofenopril or placebo.

Patients were excluded from the study if they had on admission: a) cardiogenic shock (Killip class 4), b) systolic blood pressure below $100 \mathrm{mmHg}$ (measured with the patient supine), c) serum creatinine concentration above $2.5 \mathrm{mg} / \mathrm{dL}(221 \mathrm{mmol} / \mathrm{L})$, d) history of congestive heart failure, e) current treatment with an ACE inhibitor, f) contraindications to the use of ACE inhibitors, or g) were unable or unwilling to give informed consent. All potentially eligible patients received standard therapy including analgesic agents, beta-blockers, nitrates, calcium antagonists, aspirin, inotropic drugs, diuretic agents, and anticoagulants as indicated.

The initial dose of study medication was $7.5 \mathrm{mg}$ and was repeated after 12 hours and progressively doubled up to the final dose of $30 \mathrm{mg}$ twice daily if systolic blood pressure remained above $100 \mathrm{mmHg}$ and there were no signs or symptoms of hypotension. Patients were evaluated while they were in the hospital (between day 7 and day 15 from index myocardial infarction) after 4 weeks, and at the end of the double-blind treatment period (6 weeks), during which time they could be treated with any other drug except ACE inhibitors. On completion of the 6-week double-blind period, the patients stopped taking the study medications but continued treatment in an open-label fashion for additional 11 months $( \pm 1)$ when their vital status was blindly evaluated.

The study was conducted in accordance with the Declaration of Helsinki and was approved by the institutional review board of the University of Bologna as well as by the local ethics committees when required. All patients provided written informed consent prior to inclusion into the study. Full details on the SMILE protocol are reported in Ambrosioni et al (1995) and Borghi et al (2003).

In the present post-hoc analysis a subgroup of 1418 $\mathrm{MS}+$ and MS- patients were considered. Patients were classified as having MS according to modified NCEP-ATP III criteria (NCEP ATP III 2001; Grundy et al 2004), ie, concomitant presence of at least 3 over 5 among the following risk factors: a) treated hypertension or blood pressure $\geq 130 / 85 \mathrm{mmHg}$, b) serum triglycerides $\geq 150 \mathrm{mg} / \mathrm{dL}$, c) serum HDL cholesterol $<40 \mathrm{mg} / \mathrm{dL}$ in men and $<50 \mathrm{mg} / \mathrm{dL}$ in women, d) overweight (BMI $>25 \mathrm{~kg} / \mathrm{m}^{2}$ ), and e) fasting blood glucose $\geq 110 \mathrm{mg} / \mathrm{dL}$.

\section{Study end points}

The primary study end point was the combined occurrence of death or severe congestive heart failure during the 6 weeks of treatment with zofenopril or placebo, both given in addition to conventional treatment. Death or presence of severe congestive heart failure was considered as a single event for each patient and their prevalence was calculated according to whichever occurred first.

The causes of death were classified by the principal investigators and reviewed by an end-point committee acting on the basis of a blinded review. All deaths occurring during the trial were classified as due to cardiac or non-cardiac causes. Cardiac causes included progressive heart failure, sudden death, recurrent myocardial infarction, and cardiac rupture. Non-cardiac causes included cerebrovascular events, pulmonary embolism, and non-vascular causes. Progressive heart failure was classified on the basis of pump failure and the occurrence of cardiogenic shock. Sudden death was defined 
as sudden, unexpected death occurring within 1 hour after the onset of new symptoms.

Predefined secondary end point was the effect of 6-week double-blind treatment on cumulative 1-year mortality.

\section{Statistical analysis}

The present study is a post hoc analysis of the main SMILE Study data carried out on MS+ and MS- patients. The baseline characteristics and the distribution of the various parameters for the MS+ and MS- patients were compared using a Chi-square test for categorical variables (with Yates' continuity correction where appropriate) and a standard normal t-test for continuous variables. A Chi-square test was also employed to compare the frequency of metabolic risk factors between the placebo and active treatment group. Relative risk reductions and corresponding 95\% confidence intervals $(\mathrm{CI})$ were calculated for both the primary and

Table I Baseline characteristics of study population

\begin{tabular}{|c|c|c|c|}
\hline & $\begin{array}{l}\text { MS }+ \\
(n=686)\end{array}$ & $\begin{array}{l}\text { MS- } \\
(n=732)\end{array}$ & $\mathbf{p}$ \\
\hline Age (years, means $\pm S D$ ) & $63 \pm 10$ & $65 \pm 10$ & NS \\
\hline $\begin{array}{l}\text { Gender ratio } \\
\text { (male/female, \%) }\end{array}$ & $69 / 31$ & $76 / 24$ & NS \\
\hline $\begin{array}{l}\text { Body weight } \\
(\mathrm{kg}, \text { means } \pm \mathrm{SD})\end{array}$ & $77 \pm 11$ & $70 \pm 10$ & $<0.01$ \\
\hline Current smokers (\%) & 57 & 60 & NS \\
\hline Diabetes mellitus (\%) & 30 & 15 & $<0.01$ \\
\hline $\begin{array}{l}\text { Previous myocardial } \\
\text { infarction (\%) }\end{array}$ & 18 & 17 & NS \\
\hline Hypertension (\%) & 46 & 34 & $<0.01$ \\
\hline Medication (\%) & & & \\
\hline Antiplatelet agents & 56 & 61 & NS \\
\hline Calcium channel blockers & 16 & 13 & NS \\
\hline$\beta$-blockers & 44 & 39 & NS \\
\hline Diuretics & 11 & 9 & $<0.05$ \\
\hline Nitrates & 41 & 41 & NS \\
\hline $\begin{array}{l}\text { Hours to randomization } \\
\text { (\% of patients) }\end{array}$ & & & \\
\hline$<6$ & 44 & 42 & NS \\
\hline $6-12$ & 27 & 31 & NS \\
\hline$>12$ & 29 & 27 & NS \\
\hline $\begin{array}{l}\text { Hours to randomization } \\
\text { (means } \pm S D \text { ) }\end{array}$ & $2 \pm 1$ & $2 \pm 1$ & NS \\
\hline $\begin{array}{l}\text { Hours to treatment } \\
\text { (means } \pm S D)\end{array}$ & $3 \pm 1$ & $3 \pm 1$ & NS \\
\hline $\begin{array}{l}\text { Killip class on admission } \\
>\text { I (\%) }\end{array}$ & 13 & 12 & NS \\
\hline Peak CK $\left(U^{*} 10^{-3}\right)$ & $2 \pm 1$ & $I \pm I$ & NS \\
\hline $\mathrm{SBP}(\mathrm{mmHg}$, means $\pm \mathrm{SD})$ & $143 \pm 19$ & $132 \pm 19$ & $<0.01$ \\
\hline $\mathrm{DBP}(\mathrm{mmHg}$, means $\pm \mathrm{SD})$ & $88 \pm 11$ & $82 \pm 11$ & $<0.01$ \\
\hline $\mathrm{HR}(\mathrm{mmHg}$, means $\pm \mathrm{SD})$ & $83 \pm 15$ & $81 \pm 16$ & NS \\
\hline
\end{tabular}

Abbreviations: CK, creatinine kinase; SBP, systolic blood pressure; DBP, diastolic blood pressure; HR, heart rate.
Table 2 Prevalence of the different markers of metabolic syndrome in the two randomization groups

\begin{tabular}{lll}
\hline & $\begin{array}{l}\text { Placebo } \\
(\mathbf{n}=\mathbf{6 9 9})\end{array}$ & $\begin{array}{l}\text { Zofenopril } \\
(\mathbf{n}=\mathbf{7 1 9 )}\end{array}$ \\
\hline Obesity (\%) & 32 & 32 \\
Hypertriglyceridemia (\%) & 34 & 33 \\
Low HDL cholesterol (\%) & 41 & 37 \\
Hyperglycemia & 60 & 62 \\
Hypertension (\%) & 68 & 71 \\
\hline
\end{tabular}

secondary end point, separately for the MS+ and MSpatients. The Chi-square analysis was applied to data with the Mantel-Haenzsel extension for the comparisons between the two treatment groups. Time-to-event curves were drawn using Kaplan-Meier estimates. All analyses were performed on an intention-to-treat basis and all p values are two-tailed (Norman and Streiner 2000).

\section{Results}

\section{Patients}

Of the 1418 included in this post-hoc analysis, $686(48.3 \%)$ were MS+ and 732 MS-. Placebo and zofenopril (Menarini Group, Florence, Italy) treatment was equally distributed within MS+ $(n=324$ for placebo and $n=362$ for zofenopril $)$ and MS- patients $(\mathrm{n}=375$ and $\mathrm{n}=357)$.

The MS+ and MS- groups were homogeneous for baseline clinical characteristics, except for significantly higher body weight and blood pressure values, and significantly greater prevalence of diabetes and hypertension in the MS+ group (Table 1$)$. A trend $(p=0.05)$ for a greater use of antihypertensive drugs was observed in $\mathrm{MS}+$ patients at enrolment (Table 1), but during the 6 weeks of double-blind therapy concomitant pharmacologic treatments were not different between the two treatment groups (data not shown).

As shown in Table 2, the prevalence of the different markers of the metabolic syndrome was similar in the two randomization groups. In particular hypertension and hyperglycemia were the most common risk factors and occurred in more than $50 \%$ of study population.

\section{Primary outcome measures}

During the 6 weeks of treatment, the rate of death or severe congestive heart failure was less in MS+ than in MS-patients. In MS+ patients a significant relative risk reduction of a major cardiovascular event was observed with zofenopril compared with placebo (69\%, 95\% CI: 7\%-78\%; 2p = 0.002) (Figure 1, left panel). Cumulative incidence of combined death and severe heart failure in the course of the 6 weeks of treatment 


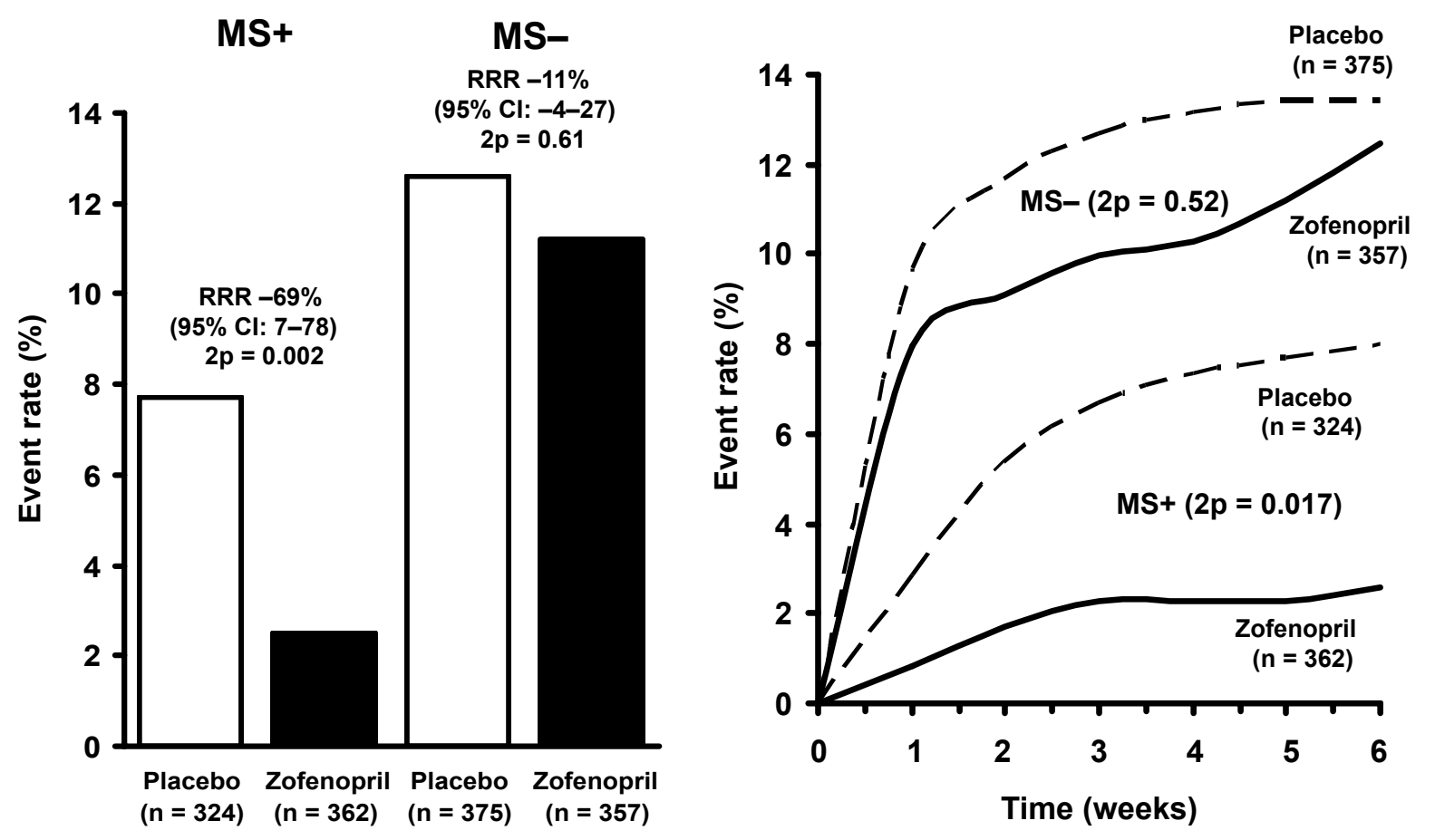

Figure I Combined occurrence of death and severe heart failure at 6 weeks (left panel) and cumulative incidence during the treatment period (right panel) in patients with (MS+) and without metabolic syndrome (MS-) treated with placebo or zofenopril.

in the MS+ group was significantly $(\mathrm{p}=0.017)$ greater under placebo than under zofenopril (Figure 1, right panel).

\section{Secondary outcome measures}

The 1-year mortality rates for the MS+ and MS- patients according to their original treatment assignments are shown in Figure 2. Patients who received zofenopril for 6 weeks were significantly more likely to survive than patients given placebo. However, risk of death was lower and relative risk reduction greater in MS+ than in MS- $(-29 \%$ vs $-19 \%$, relative risk reduction in MS+ vs MS-).

No dose-response effect between zofenopril and clinical events has been observed for either primary or secondary outcomes.

\section{Blood pressure profile}

At the end of the 6-week treatment period supine systolic and diastolic blood pressure reduction was significantly greater in MS+ than in MS- patients (Figure 3). As a consequence the final blood pressure levels achieved after 6 weeks of treatment were $129 \pm 7 / 81 \pm 9$ in MS+ and $128 \pm 8 / 78 \pm 10$ in MS- with no difference among the two populations of patients. The extent of blood pressure decrease was slightly enhanced in patients with MS and treated with zofenopril, and this difference achieved a formal statistical significance for systolic blood pressure $(\mathrm{p}<0.05)$. Heart rate was comparably reduced in both groups (data not shown).

\section{Discussion}

This post-hoc analysis of the SMILE Study (Ambrosioni et al 1995) demonstrated that early treatment with zofenopril of MS+ patients with non-thrombolyzed anterior acute myocardial infarction a) reduces the 6-week combined incidence of death and severe refractory congestive heart failure, and b) improves long-term survival after withdrawal of treatment. The relative risk reduction for the two study end points under zofenopril in MS+ patients was $69 \%$ and $29 \%$, while it was definitely lower in MS- patients $(-11 \%$ and $-19 \%$, respectively).

To our knowledge this is the first evidence from a randomized, placebo-controlled study of the prognostic benefit of early ACE inhibition in MS+ patients with acute myocardial infarction. Indeed, there are some data in the literature proving that antagonism of the renin-angiotensin system with ACE inhibitors provides substantial long-term clinical benefits in high risk patients. However, they are limited to patients with diabetes and acute myocardial infarction (Zuanetti et al 1997; Gustafsson et al 1999) or diabetes and 


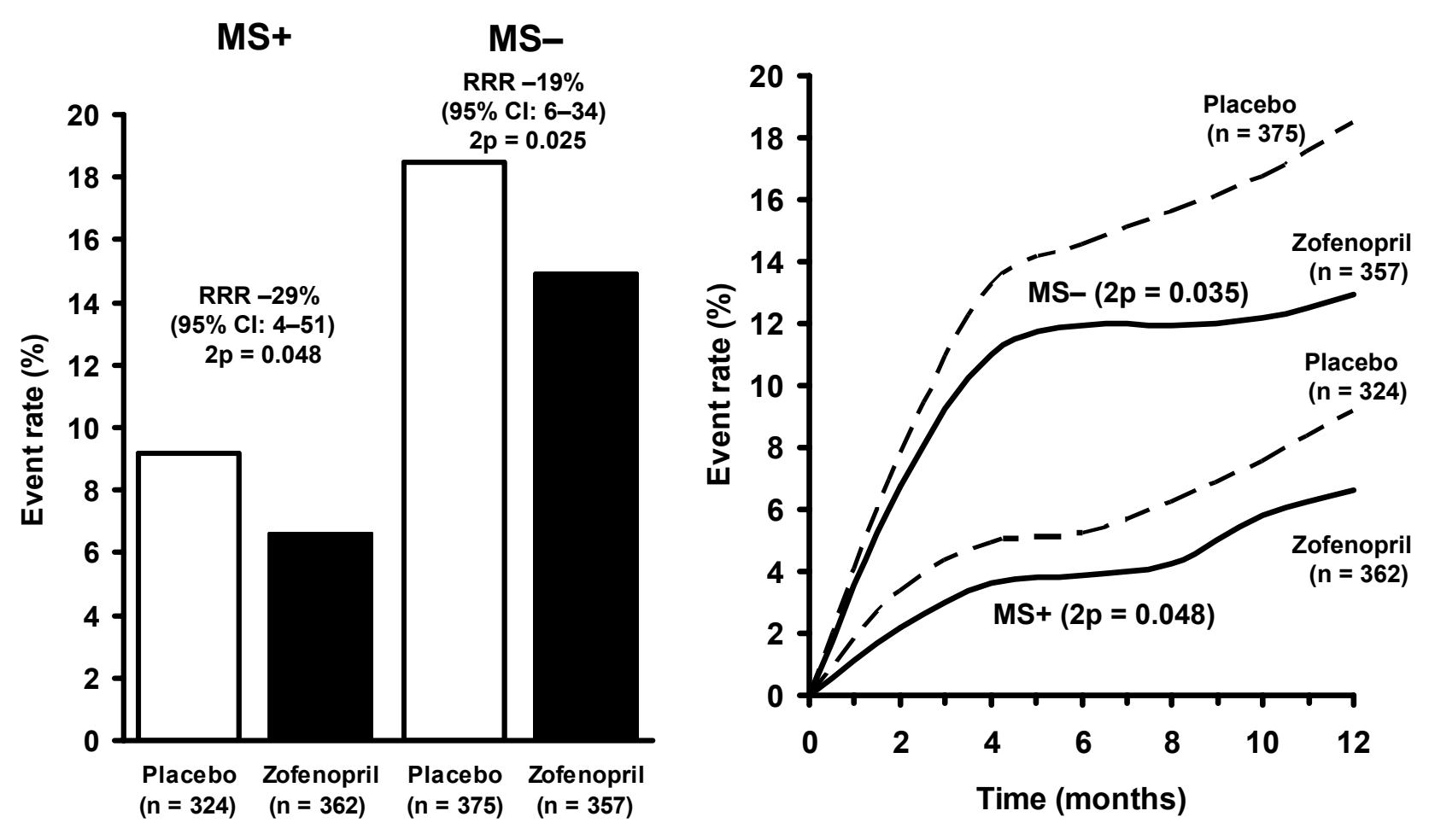

Figure 2 One-year mortality (left panel) and cumulative incidence of death (right panel) in patients with (MS+) and without metabolic syndrome (MS-) treated with placebo or zofenopril.

chronic coronary syndrome (HOPE Study Group 2000; Daly et al 2005), and also include a post-hoc analysis of the SMILE Study performed in diabetic patients (Borghi et al 2003). The relative risk reduction achieved in our study, even considering the differences in type of end points and treatment duration among the referred studies, and the fact that half our patients had hyperglycemia, exceeds that previously reported.

Our study also demonstrates that the prevalence of the MS in patients presenting at the hospital within 24 hours of an acute anterior myocardial infarction and not reperfused is high, in agreement with only two available publications on this topic (Ninomiya et al 2004; Zeller et al 2005).

Interestingly, the reduction in morbidity and mortality observed in the SMILE post-hoc study was largely independent of blood pressure reduction, suggesting that zofenopril may affect MS+ patient's prognosis beyond and in addition to its hemodynamic effect. This may be plausible, since both myocardial infarction and MS are associated with a neurohumoral activation which involves the sympathetic nervous system and the renin-angiotensin system (Walsh et al 1999). In particular in MS+ patients this activation might be also related to the condition of insulin resistance and to the consequent hyperinsulinemia that has been frequently described (Eckel et al 2005), and that could be responsible for a concentration-dependent overexpression of $\mathrm{AT}_{1}$ receptors for angiotensin II at the tissue level (Muller et al 2000). In particular in MS+ patients with myocardial infarction, this mechanism could negatively interact with the concomitant activation of circulating renin-angiotensin-system, thereby providing a reasonable explanation for the greater preventive effects of ACE inhibition observed in the SMILE Study (Prasad and Quyyumi 2004). In addition, the beneficial effects of ACE inhibition in post-myocardial infarction patients with MS can be also due to some primary protective effects of zofenopril against the deterioration of left ventricular function and the progression of the atherosclerotic disease that follows myocardial ischemia (Frascarelli et al 2004).

Some limitations of our study also deserve to be discussed. First, the study is based on a post-hoc analysis of the SMILE Study and might be underpowered to demonstrate the study goal. However, a) it was based on $91 \%$ of the original study population, b) the randomization groups were well balanced, and c) the extent of relative risk reduction observed with zofenopril was similar or even greater than that observed in high risk patients with coronary disease treated with ACE inhibitors (Gustafsson et al 1999; Zuanetti et al 1997; Moye et al 1994; HOPE Study Group 2000; Daly 


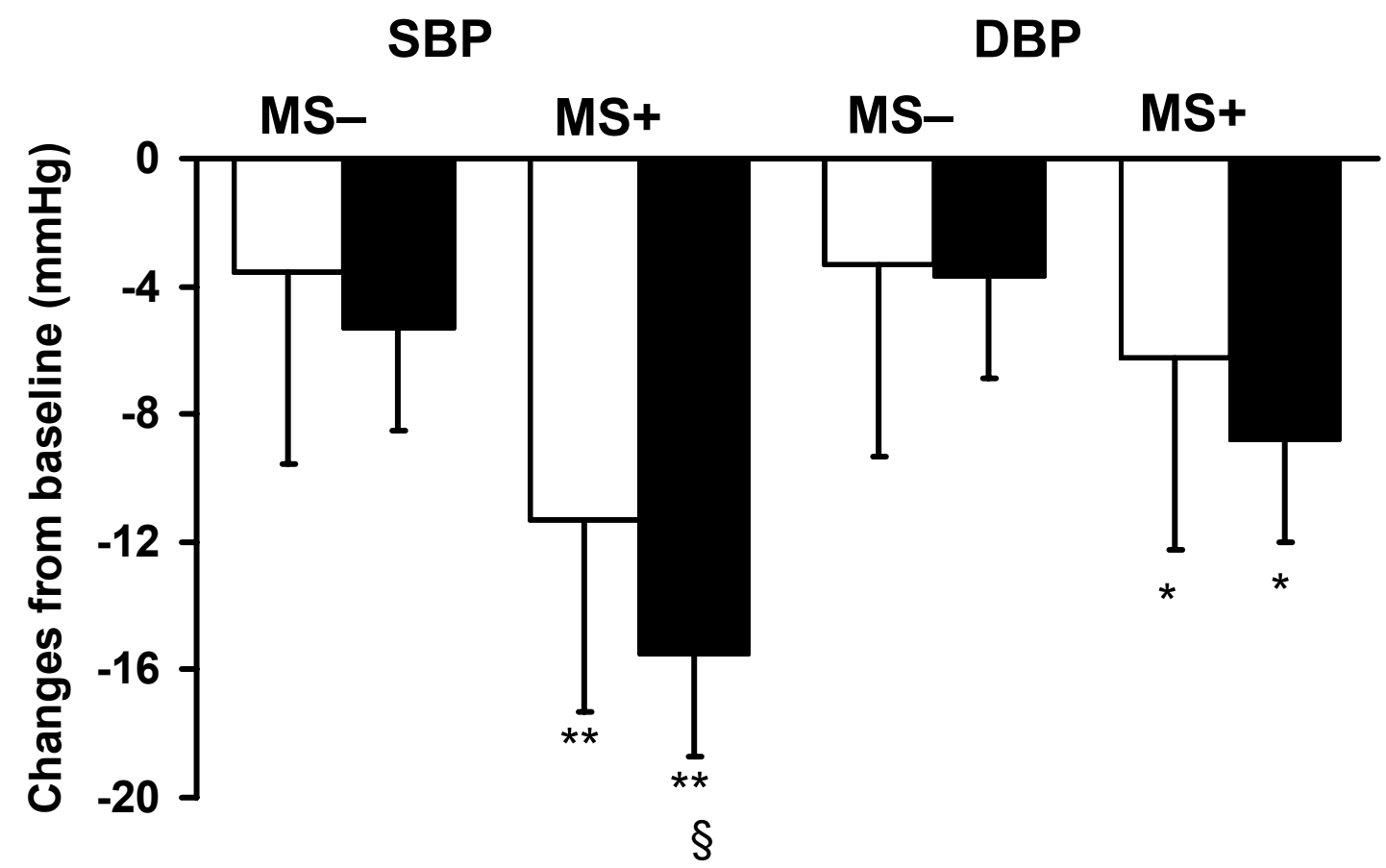

Figure 3 Changes in supine systolic blood pressure (SBP) and diastolic blood pressure (DBP) after 6 weeks of treatment with placebo (open bars, $n=699$ ) or zofenopril (full bars, $n=719)$ in patients with $(M S+, n=686)$ or without metabolic syndrome $(M S-, n=732)$. Data are shown as means \pm SD. $\left({ }^{*}, * * \mathrm{p}<0.05,0.01\right.$ vs MS-; ${ }^{5} \mathrm{p}<0.05$ vs placebo).

et al 2005). Second, this post-hoc analysis was retrospective. However, since no prospective studies on the acute benefit of ACE inhibition in MS+ patients with non-thrombolyzed acute myocardial infarction are available, we must rely on evidence provided by the retrospective data which are the only data available for analysis. Third, for the definition of MS we rely on a modified NCEP ATP III classification, ie, we used body mass index as a surrogate of waist circumference for defining abdominal obesity. Recent data suggest that the use of body mass index for the definition of MS do not negatively affect the assessment of the prevalence and prognostic impact of this condition in the general population (Hunt et al 2004). Moreover, we have a posteriori verified that this co-linearity exists in a subgroup of the cohort that is still continuously followed up.

In conclusion, results of this post-hoc analysis of the SMILE Study demonstrate the striking benefit of early administration of zofenopril in MS+ patients with acute anterior myocardial infarction and suggest the use of ACE inhibitors for the routine treatment of such patients.

\section{Acknowledgments}

Authors wish to thank Menarini group (Firenze, Italy) for the unconditioned support to the SMILE study group.

\section{Disclosures}

None of the authors has any conflicts of interest to disclose.

\section{References}

Ambrosioni E, Borghi C, Magnani B. 1995. The effect of the angiotensinconverting-enzyme inhibitor zofenopril on mortality and morbidity after anterior myocardial infarction. The Survival of Myocardial Infarction Long-Term Evaluation (SMILE) Study Investigators. $N$ Engl J Med, 332:80-5.

Borghi C, Ambrosioni E. Survival of Myocardial Infarction Long-term Evaluation-2 Working Party. 2003. Double-blind comparison between zofenopril and lisinopril in patients with acute myocardial infarction: results of the Survival of Myocardial Infarction Long-term Evaluation-2 (SMILE-2) study. Am Heart J, 145:80-7.

Borghi C, Bacchelli S, Degli Esposti D, et al. 2004. A review of the angiotensin-converting enzyme inhibitor, zofenopril, in the treatment of cardiovascular diseases. Expert Opin Pharmacother, 5:1965-77.

Borghi C, Bacchelli S, Esposti DD, et al; SMILE Study. 2003. Effects of the early ACE inhibition in diabetic nonthrombolyzed patients with anterior acute myocardial infarction. Diab Care, 26:1862-8.

Borghi C, Marino P, Zardini P, et al. 1998. Short- and long-term effects of early fosinopril administration in patients with acute anterior myocardial infarction undergoing intravenous thrombolysis: results from the Fosinopril in Acute Myocardial Infarction Study. FAMIS Working Party. Am Heart J, 136:213-225.

Daly CA, Fox KM, Remme WJ, et al; EUROPA Investigators. 2005. The effect of perindopril on cardiovascular morbidity and mortality in patients with diabetes in the EUROPA study: results from the PERSUADE substudy. Eur Heart J, 26:1369-78.

Donnelly R, Manning G. 2007. Angiotensin-converting enzyme inhibitors and coronary heart disease prevention. J Renin Angiotensin Aldosterone Syst, 8:13-22. 
Eckel RH, Grundy SM, Zimmet PZ. 2005. The metabolic syndrome. Lancet, 365:1415-28.

Ford ES, Giles WH, Dietz WH. 2002. Prevalence of the metabolic syndrome among US adults: findings from the third National Health and Nutrition Examination Survey. JAMA, 287:356-9.

Frascarelli S, Ghelardoni S, Ronca-Testoni S, Zucchi R. 2004. Cardioprotective effect of zofenopril in perfused rat heart subjected to ischemia and reperfusion. J Cardiovasc Pharmacol, 43:294-9.

Grundy SM, Cleeman JI, Merz CN, et al; Coordinating Committee of the National Cholesterol Education Program. 2004. Implications of recent clinical trials for the National Cholesterol Education Program Adult Treatment Panel III Guidelines. J Am Coll Cardiol, 44:720-32.

Gustafsson I, Torp-Pedersen C, Kober L, et al. 1999. Effect of the angiotensin-converting enzyme inhibitor trandolapril on mortality and morbidity in diabetic patients with left ventricular dysfunction after acute myocardial infarction. Trace Study Group. J Am Coll Cardiol, 34:83-9.

HOPE Study Group. 2000. Effects of ramipril on cardiovascular and microvascular outcomes in people with diabetes mellitus: results of the HOPE study and MICRO-HOPE substudy. Heart Outcomes Prevention Evaluation Study Investigators. Lancet, 355:253-9.

Hu G, Qiao Q, Tuomilehto J, et al; DECODE Study Group. 2004. Prevalence of the metabolic syndrome and its relation to all-cause and cardiovascular mortality in nondiabetic European men and women. Arch Intern Med, 164:1066-76.

Hunt KJ, Resendez RG, Williams K, et al; San Antonio Heart Study. 2004. National Cholesterol Education Program versus World Health Organization metabolic syndrome in relation to all-cause and cardiovascular mortality in the San Antonio Heart Study. Circulation, 110:1251-7.

Levantesi G, Macchia A, Marfisi R, et al; GISSI-Prevenzione Investigators. 2005. Metabolic syndrome and risk of cardiovascular events after myocardial infarction. J Am Coll Cardiol, 46:277-83.

Muller C, Reddert A, Wassmann S, et al. 2000. Insulin-like growth factor induces up-regulation of AT(1)-receptor gene expression in vascular smooth muscle cells. J Renin Angiotensin Aldosterone Syst, 1:273-7.
National Cholesterol Education Program (NCEP) Expert Panel on Detection, Evaluation, and Treatment of High Blood Cholesterol in Adults (Adult Treatment Panel III). 2002. Third Report of the National Cholesterol Education Program (NCEP) Expert Panel on Detection, Evaluation, and Treatment of High Blood Cholesterol in Adults (Adult Treatment Panel III) final report. Circulation, 106:3143-421.

Ninomiya JK, L'Italien G, Criqui MH, et al. 2004. Association of the metabolic syndrome with history of myocardial infarction and stroke in the Third National Health and Nutrition Examination Survey. Circulation, 109:42-6.

Nissen SE, Tuzcu EM, Libby P, et al. for CAMELOT Investigators. 2004. Effect of antihypertensive agents on cardiovascular events in patients with coronary disease and normal blood pressure: the CAMELOT study: a randomized controlled trial. JAMA, 292:2217-25.

Norman GR, Streiner DL. 2000. Biostatistics. The bare essentials. 2nd ed. Hamilton, Canada: B.C. Decker Inc. pp.139-44.

Prasad A, Quyyumi AA. 2004. Renin-angiotensin system and angiotensin receptor blockers in the metabolic syndrome. Circulation, 110:1507-12.

Walsh JT, Batin PD, Hawkins M, et al. 1999. Ventricular dilatation in the absence of ACE inhibitors: influence of haemodynamic and neurohormonal variables following myocardial infarction. Heart, 81:33-9.

Zeller M, Steg PG, Ravisy J, et al; Observatoire des Infarctus de Cote-d'Or Survey Working Group. 2005. Prevalence and impact of metabolic syndrome on hospital outcomes in acute myocardial infarction. Arch Intern Med, 165:1192-8.

Zhang C, Knudson JD, Setty S, et al. 2005. Coronary arteriolar vasoconstriction to angiotensin II is augmented in prediabetic metabolic syndrome via activation of AT1 receptors. Am J Physiol Heart Circ Physiol, 288:H2154-H62.

Zuanetti G, Latini R, Maggioni AP, et al. 1997. Effect of the ACE inhibitor lisinopril on mortality in diabetic patients with acute myocardial infarction: data from the GISSI-3 study. Circulation, 96:4239-45. 
